$$
\begin{array}{r}
\text { SAN097-2736C } \\
\text { SAND- } 97-2736 C
\end{array}
$$

\title{
Molybdenum Erosion Measurements in Alcator C-Mod
}

$$
\text { CONF-980560-- }
$$

W. R. Wampler*, B. LaBombard, B. Lipshultz, G. M. McCracken*,

D. Pappas and C. S. Pitcher

MIT Plasma Science and Fusion, Cambridge, MA 02139

- Sandia National Laboratories, Albuquerque, NM 87185-1056

" JET Joint Undertaking, Abingdon Oxon OX14 3EA,UK

\begin{abstract}
Erosion of molybdenum was measured on a set of 21 tiles after a run campaign of 1090 shots in the Alcator C-Mod tokamak. The net erosion of molybdenum, was determined from changes in the depth of a thin chromium marker layer measured by Rutherford backscattering. Net Mo erosion was found to be approximately $150 \mathrm{~nm}$ near the outer divertor strike point, and much less everywhere else. Gross erosion rates by sputtering were estimated using ion energies and fluxes obtained from Langmuir probe measurements of edge-plasma conditions. Predicted net ierosion using calculated gross erosion with prompt redeposition and measured net erosion agree within a factor of 3 . Sputtering by boron and molybdenum impurities dominates erosion.
\end{abstract}

DISTRIBUTION OF THS DOCUMENT Is UNLIMTED h NASTER 


\section{Introduction}

Erosion of plasma-facing structures is one of the most serious issues facing long-pulse fusion devices. However, net erosion is the combined result of complex processes including sputtering by deuterium and by heavier plasma impurities, transport of eroded material by the plasma, and redeposition. Measurements of net erosion by tokamak plasmas are therefore needed to predict long term erosion. Net erosion rates exceeding $10 \mathrm{~cm} /$ year have been measured on graphite divertor plates in DIII-D [1]. This erosion rate would be unacceptably high in a long pulse fusion reactor. One way to reduce the erosion rate is through the use of high-Z materials, which are expected to erode more slowly than low-Z materials because of their lower sputtering yields. Here we examine erosion of molybdenum in Alcator C-Mod, in which all plasma-contacting surfaces are Mo tiles. The net Mo erosion, after a run campaign of 1090 shots, was determined from changes in the depth of a thin chromium marker layer measured by Rutherford backscattering. Gross erosion by sputtering was estimated using ion energies and fluxes obtained from Langmuir probe measurements of edge-plasma conditions. Langmuir probe measurements and Mo spectroscopy indicate that erosion is dominated by the relatively few discharges with low density and high electron temperature at the divertor plate. Comparison between measured net erosion and predicted gross erosion indicates that sputtering by plasma impurities, mainly boron, must dominate Mo erosion.

\section{Experimental Method}

In these experiments, a subsurface layer of chromium was used as a depth marker to measure net erosion of Mo from the surface of tiles. The depth of the $\mathrm{Cr}$ layer beneath the surface was measured by Rutherford backscattering before and after the tiles were exposed to tokamak plasmas. A set of 21 tiles were prepared with the buried $\mathrm{Cr}$ marker layer. The tiles were first metallographically polished to prepare a smooth surface. Thin films of chromium and then molybdenum were evaporated onto the tiles over a circular region $13 \mathrm{~mm}$ in diameter at the center of each tile. RBS spectra were taken on each tile using $2.5 \mathrm{MeV}{ }^{4} \mathrm{He}$, from which it was determined that the thickness of the Cr layer was $100 \mathrm{~nm}$ and the thickness of the Mo overlayer ranged from 300 to $600 \mathrm{~nm}$. 


\section{DISCLAIMER}

This report was prepared as an account of work sponsored by an agency of the United States Government. Neither the United States Government nor any agency thereof, nor any of their employees, makes any warranty, express or implied, or assumes any legal liability or responsibility for the accuracy, completeness, or usefulness of any information, apparatus, product, or process disclosed, or represents that its use would not infringe privately owned rights. Reference herein to any specific commercial product, process, or service by trade name, trademark, manufacturer, or otherwise does not necessarily constitute or imply its endorsement, recommendation, or favoring by the United States Government or any agency thereof. The views and opinions of authors expressed herein do not necessarily state or reflect those of the United States Government or any agency thereof. 
The tiles were installed in the Alcator C-Mod tokamak and exposed to 1090 high power plasmas during the period from November 1995 to March 1996. The location of the tiles during the exposure is shown in fig. 1. The tiles were then removed from the tokamak and again examined by ion beam analysis. Figure 2 shows RBS spectra taken using $2.5 \mathrm{MeV}^{4} \mathrm{He}$ on tile 3 from the inner wall and tile 18 from the outer strikepoint. Figure 2a illustrates how the thickness of the Mo overlayer was determined from the RBS spectra. After scattering from Mo at the external surface, He ions have an energy of about $2.1 \mathrm{MeV}$ due to the kinematics of elastic scattering. He ions scattered from Mo beneath the surface reach the detector with lower energy due to the continuous energy loss of ions as they pass through material on the way in, before scattering, and back out after scattering. The dip in the spectra is due to the $\mathrm{Cr}$ marker layer. The flat-topped feature of the spectra between the dip and the surface edge is due to scattering from Mo in the overlayer. Since the stopping power of energetic ions in materials is known [2], the width of this feature gives the thickness of the Mo overlayer [3]. The decrease in width of this feature (indicated by the arrow) corresponds to a reduction in Mo overlayer thickness from 340 to $220 \mathrm{~nm}$ for this tile.

Many of the tiles had a layer of low $\mathrm{Z}$ material on the surface. This layer was examined by RBS using $2.5 \mathrm{MeV}^{4} \mathrm{He}$ and $1.7 \mathrm{MeV}^{1} \mathrm{H}$ and by nuclear reaction analysis (NRA) using the ${ }^{11} \mathrm{~B}(\mathrm{p}, \alpha)^{8} \mathrm{Be}$ reaction with an analysis beam of $650 \mathrm{keV}$ protons. These studies showed the low $\mathrm{Z}$ surface layer to consist mainly of boron. The thickness of this boron layer was determined by RBS. The step in yield in the RBS spectrum due to scattering from Mo at the surface is shifted down in energy because of energy loss by the ions as they pass through the boron layer, as indicated by the arrow in fig. $2 \mathrm{~b}$. Using the known stopping power of ions in boron [2], the thickness of the boron layer is obtained from the magnitude of this energy shift. The boron is present because the vessel was boronized several times during the run campaign. Boron was deposited by decomposition of diborane in a plasma to reduce plasma impurities.

Nuclear reaction analysis was also used to measure deuterium (D) on the tiles. This measurement used the ${ }^{3} \mathrm{He}(\mathrm{d}, \mathrm{p}) \alpha$ nuclear reaction with an analysis beam of $700 \mathrm{keV}{ }^{3} \mathrm{He}$. The areal density of $\mathrm{D}$ within about 1 micron of the surface was determined from the yield of energetic protons. 


\section{Experimental Results}

The results from ion beam analysis of the tiles are summarized in fig. 3. The net Mo erosion was highest on tiles 17-20 at the outer strikepoint (OSP). Here the erosion was 100 to $170 \mathrm{~nm}$. All other tiles had much less Mo erosion. The thickness of the boron surface layer is also shown in fig. 3. The OSP had the least boron. Boron coverage was also relatively low at the inner strikepoint. Elsewhere, boron coverages were higher, ranging up to $1.8 \times 10^{19}$ atoms $/ \mathrm{cm}^{2}$, which corresponds to a physical thickness of $1.4 \mu \mathrm{m}$ assuming the density 2.34 $\mathrm{g} / \mathrm{cm}^{3}$ of elemental boron. On some of the tiles (tile numbers $4,5,7,8,9,10,14,15,21$ ) the boron layer was found to have nonuniform thickness over the region of the analysis beam spot $(1 \times 1 \mathrm{~mm})$. This caused a broadening of the edges in the RBS spectra used to determine the thickness of both the boron and Mo layers and a corresponding reduction in the precision of the value obtained for the thickness of these layers. The larger error bars on the boron thickness and Mo erosion shown in fig. 3 for these tiles are due to this effect.

Figure 3 shows the deuterium coverage on the tiles. The error bars for these data indicate the range of values measured at several locations on each tile. The OSP had the least $\mathrm{D}$ which is consistent with results from Mo erosion and $\mathrm{B}$ deposition which indicate that the OSP is a region undergoing net erosion of material.

$\mathrm{B}$ and $\mathrm{D}$ coverages on the nose tile of the inner strikepoint (between tiles 10 and 11 in fig. 1) were measured and are plotted in fig. 3 at tile number 10.5. However, this tile did not have the buried $\mathrm{Cr}$ depth marker and so the Mo erosion could not be determined for this tile.

\section{Calculated Erosion based on Physical Sputtering}

The outer divertor plate is instrumented with built-in Langmuir probes, which are able to deduce the incident plasma particle flux density, local plasma density and electron temperature. These probes were operated routinely throughout every shot of the campaign in which the tiles were exposed. From the probe measurements it is possible to make estimates of the gross erosion on the tiles, integrated over the campaign. The results are shown in Fig. 4. We assume physical sputtering is the dominant erosion mechanism, including sputtering by the background fuel ions $\mathrm{D}^{+}$, the dominant impurity boron and some amount of molybdenum self-sputtering. In the case of boron, a helium-like $\mathrm{B}^{+3}$ charge-state is assumed. Normal incidence sputtering yields for $\mathrm{D}$ and $\mathrm{B}$ are used, since numerical studies indicate 
only a weak dependence on angle of incidence [4]. This is in contrast to molybdenum, where a relatively strong angular dependence exists at energies near the threshold for sputtering [5]. In this case, we use the sputtering yield at 18 degrees off-normal incidence, as this angle is roughly the average angle of incidence for promptly redeposited molybdenum ions [6]. The incident ion energy is determined using,

$$
E_{0}=(2+3 * Z) T_{e}
$$

while the sputtered influx density is given by

$$
\Phi_{\text {in }}=\left(\mathrm{j}_{\mathrm{s}} / \mathrm{e}\right) * \sin (\theta) *\left(\mathrm{Y}_{\mathrm{D}}+\mathrm{a} \mathrm{Y}_{\mathrm{B}}\right) /\left(1-\mathrm{Y}_{\mathrm{Mo}}\right),
$$

[7] where $\mathrm{j}_{\mathrm{s}}$ is the parallel field ion saturation current measured by the probes, $\theta$ is the glancing field angle, $\mathrm{Y}_{\mathrm{D}, \mathrm{B}, \mathrm{Mo}}$ are the sputtering yields for $\mathrm{D}^{+}, \mathrm{B}^{+3}$ and $\mathrm{Mo}^{+}$, and $\mathrm{a}$ is the boron fraction, $\mathrm{a} \sim 0.015$.

The gross erosion as deduced from the Langmuir probe data has been compared at given times throughout the campaign with the intensity of Mo I light emission measured with a multi-chord visible light spectrometer. Good agreement is generally obtained, leading to confidence that the campaign-integrated calculations are accurate. The gross erosion is dominated by impurity sputtering, with boron and molybdenum self-sputtering being comparable. At no point in the distribution is $\mathrm{D}^{+}$sputtering significant. Gross erosion is dominated by the relatively few discharges with low density and high electron temperature at the divertor plate. The gross sputtering is highly peaked in the vicinity of the separatrix, as expected since this location has on average both elevated incident particle flux, as well as elevated particle energies.

To compare gross erosion estimated from Langmuir probe data with the net erosion measured by the surface marker technique, an estimate of redeposition is required. We have assumed only prompt redeposition, where sputtered Mo atoms, depending on the local plasma conditions, can be ionized at a distance less than one gyro-radius from the plate and thus have a significant chance of being promptly redeposited. Those particles which are not promptly redeposited in this way are assumed to be lost to the plasma, of course ultimately returning to a surface at some point in the vessel, but not strongly influencing the net erosion at the outer plate. We use the simple analytic results from Naujoks et. al. [8] to determine the prompt redeposition probability. The probe-deduced campaign-integrated gross and net erosion are 
shown in Fig. 4, along with the marker determined net erosion. In general, good agreement is obtained between the probe result and the marker erosion, both in shape and absolute magnitude.

The shape of the net erosion pattern in Fig. 4 is markedly different than the gross erosion, the latter being less peaked and peaking away from the separatrix, in contrast to the gross erosion. This is a result of strong prompt redeposition in the vicinity of the separatrix, and relatively weaker redeposition further out in the SOL, i.e. molybdenum sputtered further out in the SOL has a lower probability of being promptly redeposited due to the lower values of plasma density and temperature, and hence longer mean-free path for ionization, at this location. This results in a ratio of net to gross erosion of $\sim 0.5$ here, compared to $\sim 0.05$ near the separatrix. The calculation of erosion from Langmuir probe measurements is described in more detail in reference 9.

\section{Conclusions}

Net Mo erosion after exposure to 109 plasmas was found to be approximately $150 \mathrm{~nm}$ near the outer divertor strike point, and much less everywhere else. A surface layer of boron from boronization was found at all locations except near the OSP. Gross erosion rates by sputtering were estimated using ion energies and fluxes obtained from Langmuir probe measurements of edge-plasma conditions. Predicted net erosion using calculated gross erosion with prompt redeposition agrees with measured net erosion within a factor of three. This agreement supports the model that the principal mechanism for Mo erosion is physical sputtering by ions. Ion energies from Langmuir probe measurements and corresponding sputtering yields lead to the conclusion that erosion is mainly due to sputtering by boron and molybdenum impurities.

Sandia is a multiprogram laboratory operated by Sandia Corporation, a Lockheed Martin Company, for the United States Department of Energy under contract DE-ACO4-94AL85000. 


\section{Figure Captions}

1. Diagram of Alcator C-Mod showing the location of the 21 tiles with the $\mathrm{Cr}$ depth marker.

2. RBS spectra taken with $2.5 \mathrm{MeV}^{4} \mathrm{He}$ before (filled circles) and after (open circles) exposure to $\mathrm{C}$-mod plasmas.

3. Results from ion-beam analysis. Measured net Mo erosion (bottom), thickness of surface boron layer (center) and areal density of deuterium (top).

4. Gross Mo erosion (open symbols) calculated using Langmuir probe data, versus distance below the C-Mod midplane. Also shown is the net erosion calculated using the gross erosion with prompt redeposition (filled squares) and measured using the $\mathrm{Cr}$ depth marker (filled circles) 


\section{References}

1. D. Whyte et.al. ,J. Nucl. Mater. 241-243 (1997) 660 and this conference.

2. J. F. Ziegler, The Stopping and ranges of ions in Matter, Vol. 4, Pergamon Press, New York, NY, 1977.

3. J. R. Tesmer and M. Nastasi, editors, Handbook of Modern Ion Beam Analysis, Materials Research Society, Pittsburgh, PA, 1995.

4. Sputtering yield vs angle of incidence for D \& B

5. Sputtering yield vs angle of incidence for Mo

6. Average angle of incidence for promptly redeposited $\mathrm{Mo}=18 \mathrm{deg}$.

7. Ref for incident ion energy and influx of sputtered Mo

8. Naujoks, probability for prompt redeposition

9. D. Papas et. al., this conference 


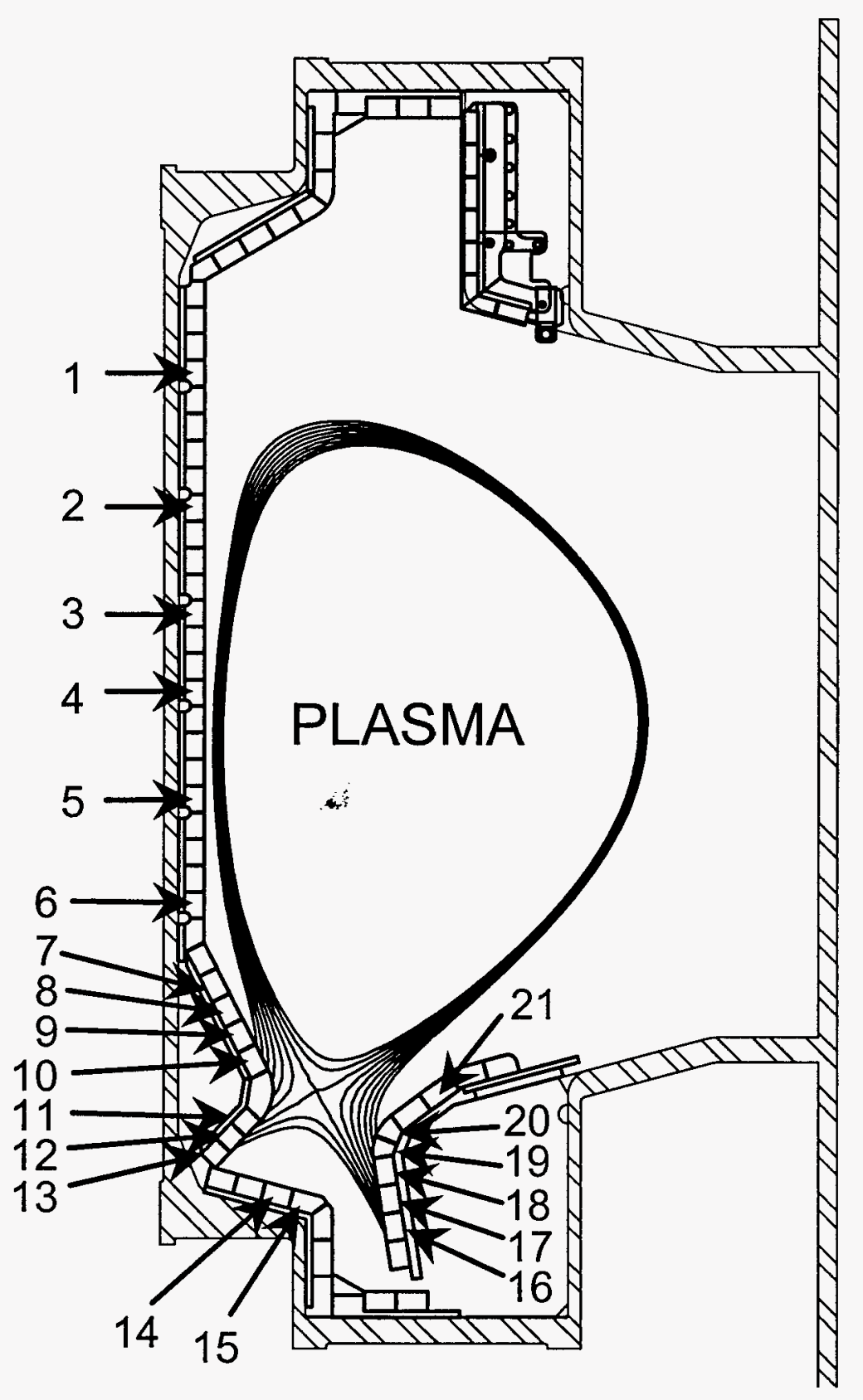



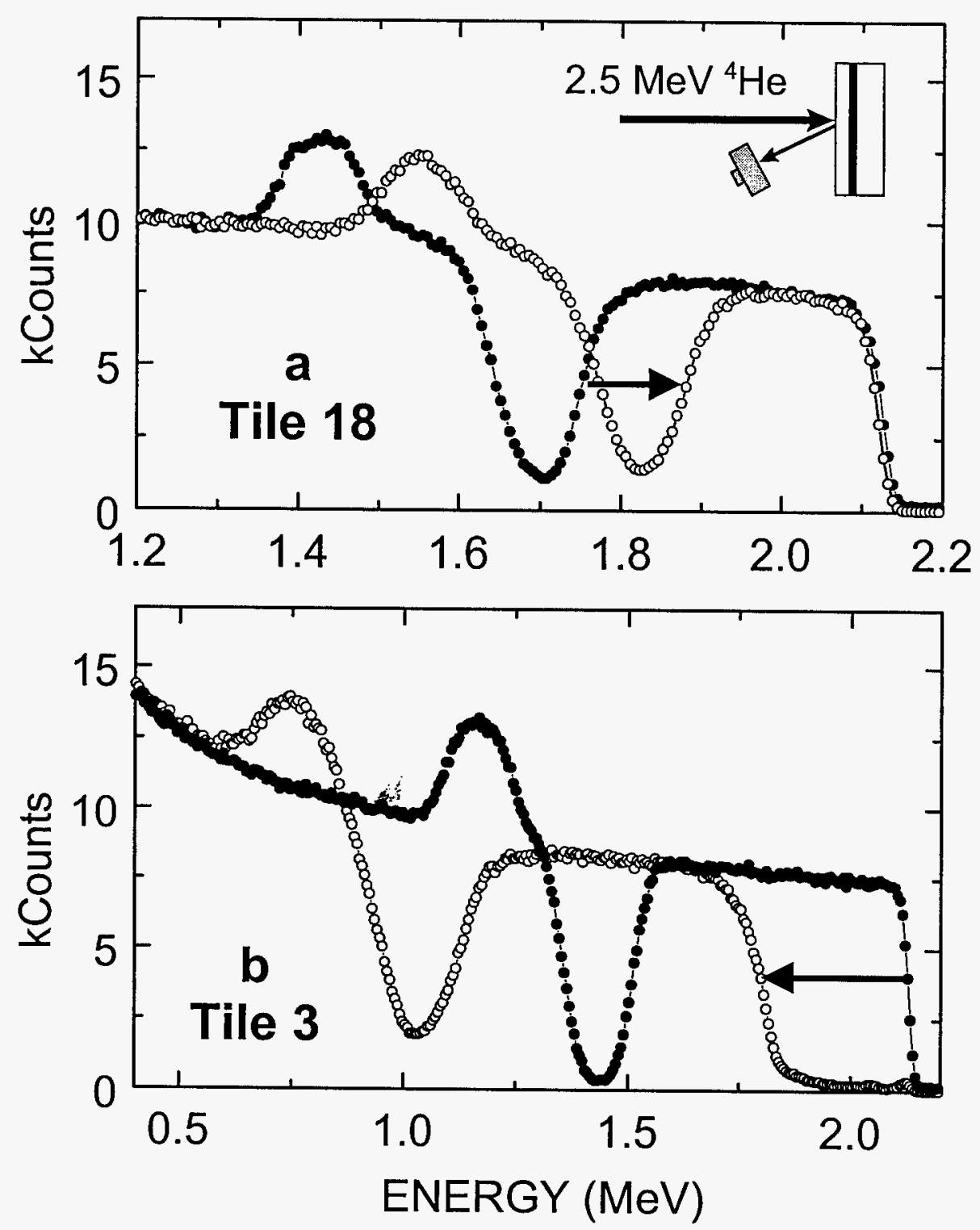

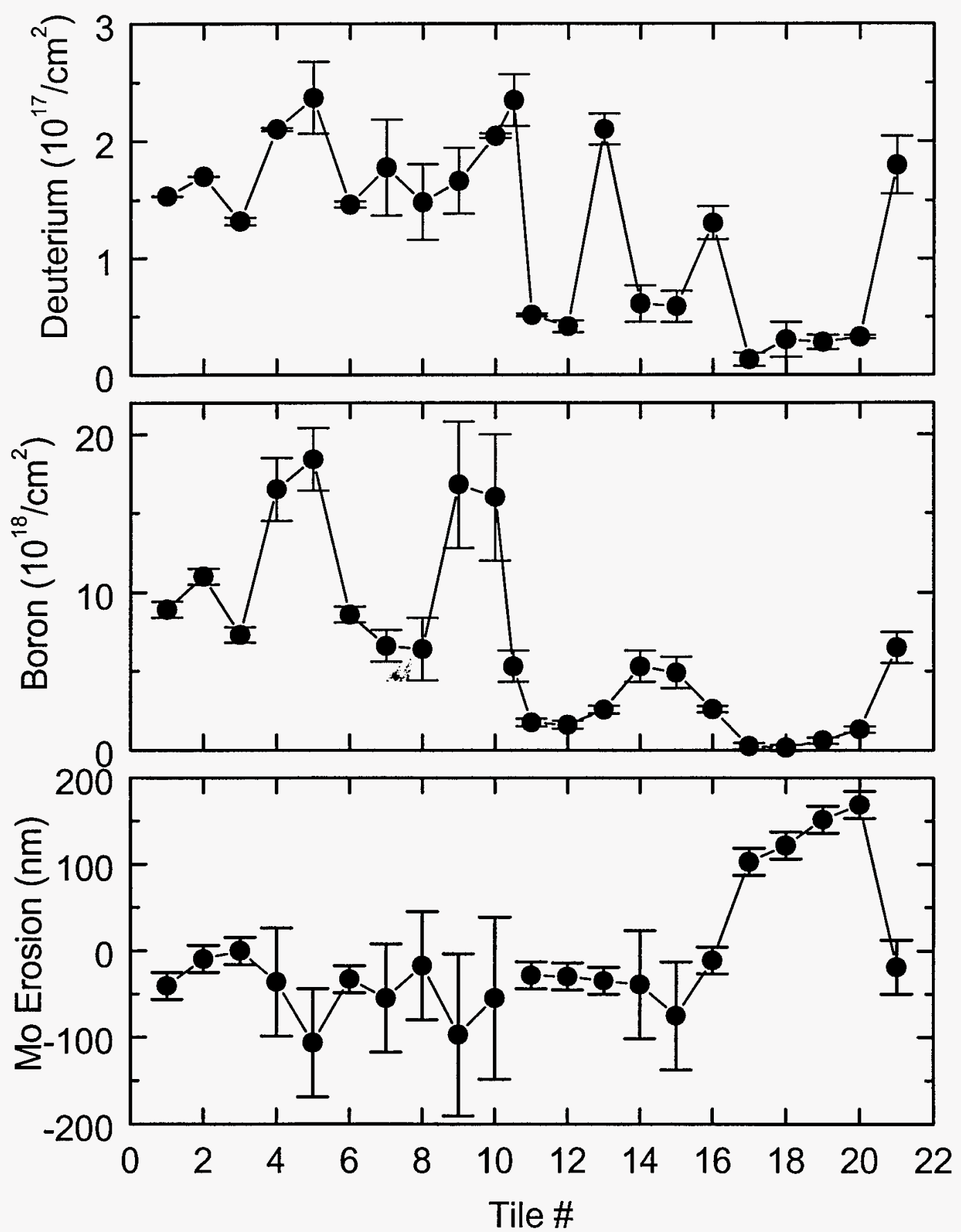


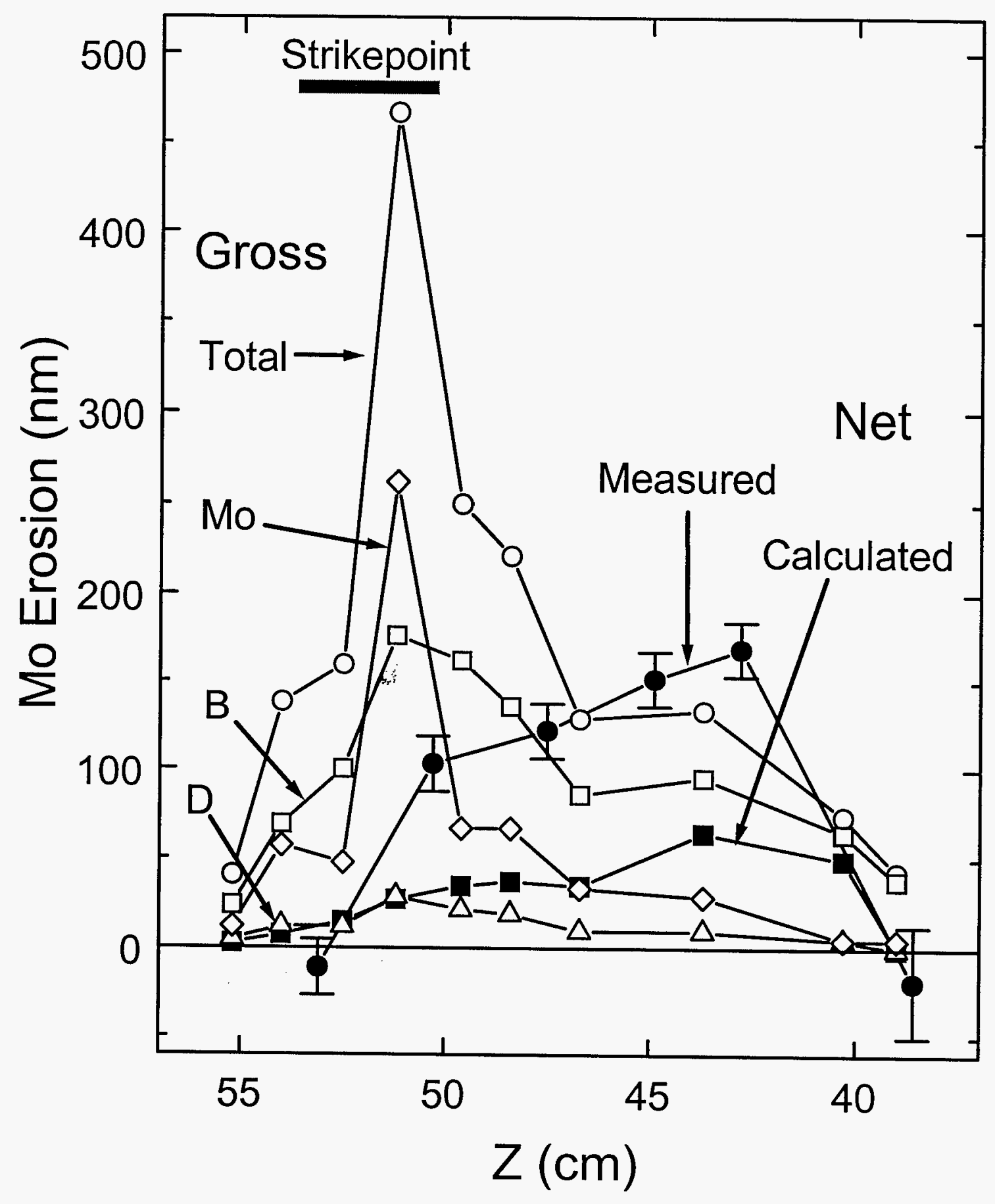




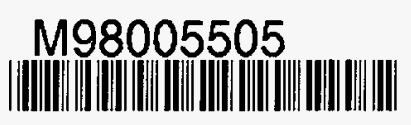

Report Number (14) $\frac{S A D D-97-2736 \mathrm{C}}{\operatorname{CONF}-950560 \cdots}$

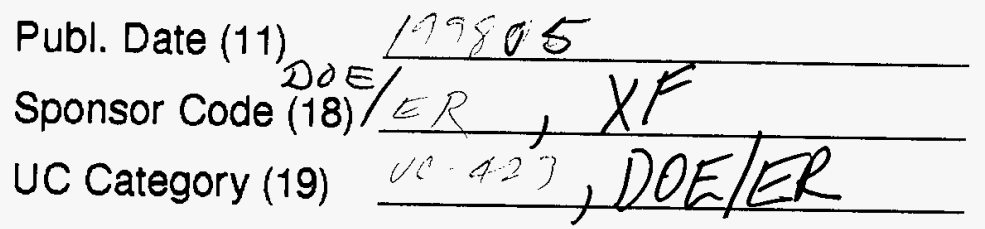

\section{3}

DTL QUALTTY INOPECTED 1

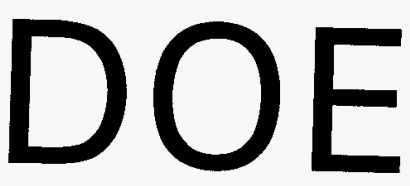

